CERN-TH/99-37

hep-th/9902125

\title{
On running couplings in gauge theories from type-IIB supergravity
}

\author{
A. Kehagias and K. Sfetsos \\ Theory Division, CERN \\ CH-1211 Geneva 23, Switzerland \\ kehagias, sfetsos@mail.cern.ch
}

\begin{abstract}
We construct an explicit solution of type-IIB supergravity describing the strong coupling regime of a non-supersymmetric gauge theory. The latter has a running coupling with an ultraviolet stable fixed point corresponding to the $\mathcal{N}=4 S U(N)$ super-Yang-Mills theory at large $N$. The running coupling has a power law behaviour, argued to be universal, that is consistent with holography. Around the critical point, our solution defines an asymptotic expansion for the gauge coupling beta-function. We also calculate the first correction to the Coulombic quark-antiquark potential.
\end{abstract}

CERN-TH/99-37

February 1999 


\section{Introduction and computations}

One of the well-known vacua of the type-IIB supergravity theory is the $A d S_{5} \times S^{5}$ one, first described in [1]. The non-vanishing fields are the metric and the anti-self-dual fiveform $F_{5}$. The latter is given by the Freund-Rubin-type ansatz, which is explicitly written as

$$
\begin{aligned}
F_{\mu \nu \rho \kappa \lambda} & =-\frac{\sqrt{\Lambda}}{2} \epsilon_{\mu \nu \rho \kappa \lambda}, \quad \mu, \nu, \ldots=0,1, \ldots, 4, \\
F_{i j k p q} & =\frac{\sqrt{\Lambda}}{2} \epsilon_{i j k p q}, \quad i, j, \ldots=5, \ldots, 9,
\end{aligned}
$$

and is clearly anti-self-dual. This background has received a lot of attention recently because of its conjectured connection to $\mathcal{N}=4 S U(N)$ super-Yang-Mills (SYM) theory at large $N$ [2, 3]. The SYM coupling $g_{\mathrm{YM}}$ is given, in terms of the dilaton $\Phi$, as $g_{\mathrm{YM}}^{2}=$ $4 \pi e^{\Phi}$, and the 't Hooft coupling is $g_{\mathrm{H}}^{2}=g_{\mathrm{YM}}^{2} N$, where $N=\int_{S^{5}} F_{5}$ is the flux of the five-form through the $S^{5}$. The dilaton is constant in this background, which is related to the finiteness of the $\mathcal{N}=4$ SYM theory.

In order to make contact with QCD, it is important to investigate deformations of the SYM theory that break conformal invariance and supersymmetry. In this case, the couplings are running corresponding to a non-constant dilaton in the supergravity side. It is then clear that the background we are after is a perturbation of $A d S_{5} \times S^{5}$. Attempts to find supergravity backgrounds that allow a non-constant dilaton, and hence a running coupling of the YM theory, have been exploited within type-0 theories [4]-[6]. Deformations of the $\mathcal{N}=4$ theory, which flow to an interacting conformal fixed point, have been considered in [7].

The purpose of the present paper is to show that running couplings are also possible within the type-IIB string theory. We will study the "minimal" case, that is we will keep the same $F_{5}$ form as in (1) and turn on a non-constant dilaton. We will show that such a solution, which breaks supersymmetry and conformal invariance, exists. We will assume for the metric four-dimensional Poincaré invariance $I S O(1,3)$, since we would like a gauge theory defined on Minkowski space-time. In addition, we will preserve the original $S O(6)$ symmetry of the $A d S_{5} \times S^{5}$. As a result, the $I S O(1,3) \times S O(6)$ invariant ten-dimensional metric is of the form ${ }^{1}$

$$
d s^{2}=g_{\mu \nu} d x^{\mu} d x^{\nu}+g_{i j} d x^{i} d x^{j}
$$

where

$$
g_{\mu \nu} d x^{\mu} d x^{\nu}=\Omega^{2}(\rho)\left(d \rho^{2}+d x_{\alpha} d x^{\alpha}\right), \quad \alpha=0,1,2,3,
$$

and $g_{i j}$ is the metric on $S^{5}$. The dilaton, by $I S O(1,3) \times S O(6)$ invariance, can only be a function of $\rho$. The supergravity equations turn out to be

$$
R_{\mu \nu}=-\Lambda g_{\mu \nu}+\frac{1}{2} \partial_{\mu} \Phi \partial_{\nu} \Phi
$$

\footnotetext{
${ }^{1}$ Supersymmetric solutions to type-IIB supergravity which, however, do not preserve the Poincaré invariance in the brane world-volume have been found in 8 .
} 


$$
\frac{1}{\sqrt{-G}} \partial_{\mu}\left(\sqrt{-G} G^{\mu \nu} \partial_{\nu} \Phi\right)=0
$$

and

$$
R_{i j}=\Lambda g_{i j} .
$$

The above equation is automatically solved for a five-sphere of radius $2 / \sqrt{\Lambda}$ and a first integral of the dilaton equation in (3) is

$$
\partial_{\rho} \Phi=A \Omega^{-3}
$$

where $A$ is a dimensionful integration constant. Moreover, the non-zero components of the Ricci tensor for the metric (2) are

$$
\begin{aligned}
& R_{\rho \rho}=-4 \partial_{\rho}^{2} \ln \Omega, \\
& R_{\alpha \beta}=-\eta_{\alpha \beta}\left(\partial_{\rho}^{2} \ln \Omega+3\left(\partial_{\rho} \ln \Omega\right)^{2}\right),
\end{aligned}
$$

and the first equation in (3) reduces to solving

$$
\left(\partial_{\rho} \ln \Omega\right)^{2}=\frac{A^{2}}{24} \Omega^{-6}+\frac{\Lambda}{4} \Omega^{2} .
$$

The solution of the above equation for $\Omega$ as a function of $\rho$ is given implicitly, in terms of a hypergeometric function, by

$$
\Omega^{3} F\left(\frac{1}{2}, \frac{3}{8}, \frac{11}{8} ;-\frac{6 \Lambda}{A^{2}} \Omega^{8}\right)= \pm \sqrt{\frac{3}{8}}|A|\left(\rho-\rho_{0}\right),
$$

where $\rho_{0}$ is another constant of integration. The different overall signs in the right-hand side of (8) arise from taking the square root in (7). We impose the boundary condition that the space described by (2) becomes $A d S_{5}$ when $\rho \rightarrow 0^{+}$. That means that the conformal factor should assume the form $\Omega \simeq R / \rho$ for small $\rho$, where $R \equiv\left(4 \pi g_{s} N\right)^{1 / 4}$. Using well-known formulae for the hypergeometric functions, we see that this naturally leads to the choice of the minus sign in (8) and also fixes $\Lambda=4 / R^{2}$. In addition, the constants $A$ and $\rho_{0}$ are related by

$$
|A|=R^{3} \frac{\eta^{4}}{\rho_{0}^{4}}, \quad \eta \equiv \sqrt{\frac{3}{8 \pi}} \frac{\Gamma(3 / 8) \Gamma(1 / 8)}{24^{3 / 8}} \simeq 1.87 .
$$

Then (8) can be written as

$$
\left(\frac{\Omega \rho_{0}}{R}\right)^{3} F\left(\frac{1}{2}, \frac{3}{8}, \frac{11}{8} ;-\frac{24}{\eta^{8}}\left(\frac{\Omega \rho_{0}}{R}\right)^{8}\right)=\sqrt{\frac{3}{8}} \eta^{4}\left(1-\frac{\rho}{\rho_{0}}\right) .
$$

We also find that

$$
\Omega \simeq(3 / 8)^{1 / 6} \eta^{4 / 3} \frac{R}{\rho_{0}}\left(1-\frac{\rho}{\rho_{0}}\right)^{1 / 3}, \quad \text { as } \quad \rho \rightarrow \rho_{0}^{-} .
$$


We may solve the dilaton equation in (5) close to $\rho=0$ and $\rho=\rho_{0}$. The result for the string coupling is

$$
e^{\Phi}=g_{s}\left(1+s \frac{\eta^{4}}{4}\left(\frac{\rho}{\rho_{0}}\right)^{4}+\frac{\eta^{8}}{32}\left(\frac{\rho}{\rho_{0}}\right)^{8}+s \frac{11 \eta^{12}}{3456}\left(\frac{\rho}{\rho_{0}}\right)^{12}+\ldots\right), \quad \text { as } \quad \rho \rightarrow 0^{+}
$$

where $s= \pm 1=\operatorname{sign}(A)$. 2 while on the other side

$$
\Phi=\Phi_{0}-s(8 / 3)^{1 / 2} \ln \left(1-\frac{\rho}{\rho_{0}}\right), \quad \text { as } \rho \rightarrow \rho_{0}^{-} .
$$

The form of the above solution is dictated by the fact that, in the limit $\rho_{0} \rightarrow \infty$, the dilaton should be $e^{\Phi}=g_{s} \equiv e^{\Phi_{0}}$ and (12) and (13) should coincide. Now, at $\rho=\rho_{0}$ there is a singularity that may be easily seen by computing the Ricci scalar using (3). The latter is $\mathcal{R}=\frac{1}{2} \partial_{\mu} \Phi \partial^{\mu} \Phi$, which at $\rho \simeq \rho_{0}$ behaves as $\mathcal{R} \sim\left(\rho_{0}-\rho\right)^{-8 / 3}$. Hence, we may consider our solution only as an asymptotic expansion around the $A d S_{5}$ geometry at $\rho=0$ of the form

$$
\Omega(\rho)=\frac{R}{\rho}\left(1+\sum_{n=1}^{\infty} a_{n}\left(\frac{\rho}{\rho_{0}}\right)^{8 n}\right), \quad \rho<\rho_{0},
$$

where the coefficients $a_{n}$ are computed using the series representation for the hypergeometric function in (10) for large $\Omega$. The first coefficient of the expansion turns out to be $a_{1}=-\eta^{8} / 432 \simeq-0.352$. Hence, $\Omega(\rho)$ is given by

$$
\Omega(\rho) \simeq \frac{R}{\rho}\left(1-\frac{\eta^{8}}{432}\left(\frac{\rho}{\rho_{0}}\right)^{8}\right)
$$

to a very good approximation, except when $\rho$ takes values very close to $\rho_{0}$. Then we may use, to a very good approximation as well, (11) instead of (15), and the results are plotted in fig. 1. Note that our analysis was done in the Einstein frame and it is not difficult to translate everything into the string frame by multiplying the Einstein metric by $e^{\Phi / 2}$. Our solution is singular at $\rho=\rho_{0}$ in both frames and can, indeed, be trusted away from that point.

Note that, the metric (2) with the conformal factor $\Omega$ specified by (10) can be written in horospherical coordinates ${ }^{3}\left(r, x^{\alpha}\right)$ determined by $\Omega(\rho) d \rho=d r$, as

$$
g_{\mu \nu} d x^{\mu} d x^{\nu}=d r^{2}+K(r) d x_{\alpha} d x^{\alpha},
$$

where

$$
K(r)=\sqrt{2} R^{2} e^{-2 r_{0} / R} \sinh ^{1 / 2}\left(\frac{4\left(r_{0}-r\right)}{R}\right) .
$$

\footnotetext{
${ }^{2}$ The solutions corresponding to the two possible choices for $s$ are related by an $S$-duality transformation and correspond to different gauge theories. This reflects the fact that, except for $\rho_{0}=\infty$, corresponding to the $\mathcal{N}=4 S U(N)$ SYM at large $N$, our solution describes gauge theories that are not $S$-duality-invariant. Also in (12) we have used (14) below.

${ }^{3}$ We thank A.A. Tseytlin for comments on this point. Also, a similar solution to that in the present paper, but with no analogous interpretation, has been given in [9].
} 
In the same coordinate system the string coupling takes the form

$$
e^{\Phi}=g_{s}\left(\operatorname{coth}\left(\frac{2\left(r_{0}-r\right)}{R}\right)\right)^{2 s a} \cdot \quad a \equiv \sqrt{6} / 4 .
$$

In the rest of the paper we prefer to work in the $\left(\rho, x^{\alpha}\right)$ coordinate system.

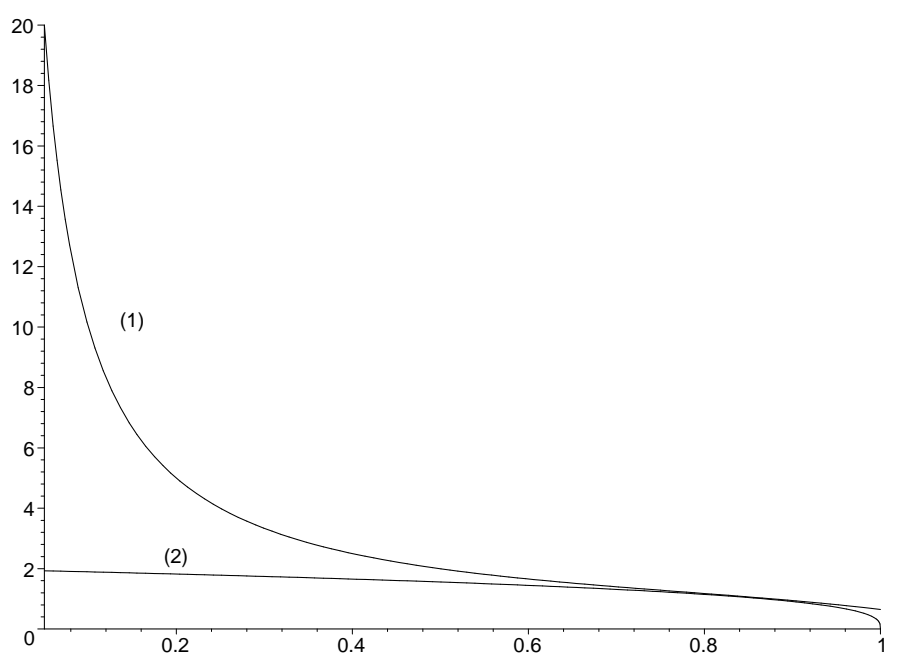

Figure 1: Plot of $\Omega(\rho) / R$ in units where $\rho_{0}=1$. Curves (1) and (2) were plotted using (15) and (11) respectively. The curve corresponding to $\Omega(\rho) / R$, obtained by numerically solving (8), coincides with the union of these curves.

\section{Running Coupling}

In the AdS/CFT scheme, the dependence of the bulk fields on the radial coordinate $\rho$ may be interpreted as energy dependence. In fact, it is a general feature in the AdS/CFT scheme that long (short) distances in the AdS space correspond to high (low) energies in the CFT [2, 10]. In particular, if the dilaton in the supergravity side is a function of $\rho$, then the 't Hooft coupling of the boundary CFT has an energy dependence and can be interpreted as the running coupling of the CFT. Running coupling means of course that we are away from conformality; thus, backgrounds that admit non-constant dilaton correspond to non-conformal field theories. As long as supersymmetry is unbroken, spinsum rules for the AdS supersymmetry are expected to protect the 't Hooft coupling $g_{\mathrm{H}}$ of the boundary $\mathcal{N}=4 \mathrm{YM}$ theory against running. However, if supersymmetry is broken, there are then no more cancellations between fermionic and bosonic contributions leading to the running of $g_{\mathrm{H}}$. The specific background we found here clearly breaks 
supersymmetry, and

$$
\begin{aligned}
\delta \lambda & =\frac{1}{2} \gamma^{M} \partial_{M} \Phi \epsilon^{*}=\frac{A}{2 \Omega^{3}} \gamma^{\rho} \epsilon^{*} \\
\delta \psi_{M} & =D_{M} \epsilon
\end{aligned}
$$

are the associated fermionic zero modes. If we now follow the correspondence between long-distances/high-energies in the AdS/CFT scheme, we find that the dual theory of the supergravity solution we obtained has a coupling with power-law running. Indeed, by changing the variable $\rho=R^{2} / U$ and interpreting $U$ as the energy of the boundary field theory, we find from (12) the running of $g_{H}$ :

$$
g_{\mathrm{H}}=g_{\mathrm{H}}^{*}\left(1+s \frac{\eta^{4}}{8} \frac{R^{8}}{\rho_{0}^{4} U^{4}}+\frac{\eta^{8}}{128} \frac{R^{16}}{\rho_{0}^{8} U^{8}}+s \frac{17 \eta^{12}}{27648} \frac{R^{24}}{\rho_{0}^{12} U^{12}}+\ldots\right),
$$

where $g_{\mathrm{H}}^{*}=R^{2}$ is the UV value of the 't Hooft coupling (the result in plotted in fig. 2). From this expression, it may easily be found that the behaviour of the beta-function for

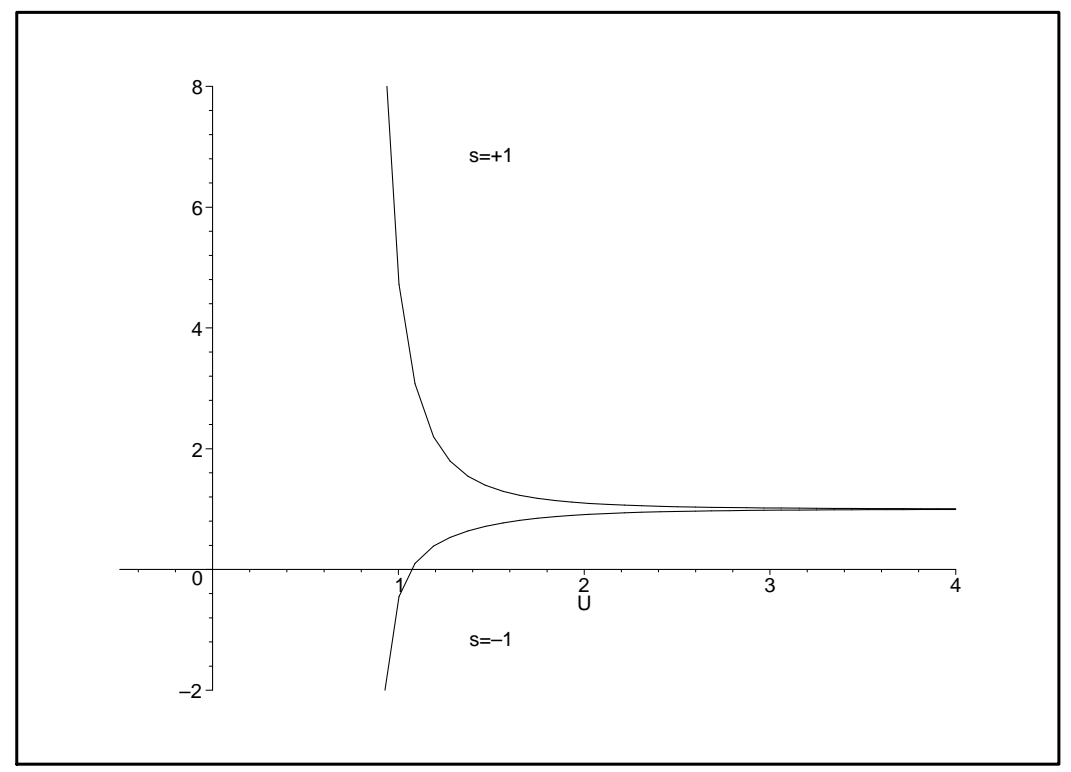

Figure 2: Plot of $g_{\mathrm{H}} / g_{\mathrm{H}}^{*}$ as a function of $U$ using (20).

the 't Hooft coupling around $g_{\mathrm{H}}^{*}$ is

$$
U \frac{d g_{\mathrm{H}}}{d U}=-4\left(g_{\mathrm{H}}-g_{\mathrm{H}}^{*}\right)-2\left(g_{\mathrm{H}}-g_{\mathrm{H}}^{*}\right)^{2} / g_{\mathrm{H}}^{*}-\frac{14}{27}\left(g_{\mathrm{H}}-g_{\mathrm{H}}^{*}\right)^{3} / g_{\mathrm{H}}^{* 2}+\mathcal{O}\left(g_{\mathrm{H}}-g_{\mathrm{H}}^{*}\right)^{4} .
$$

However, the above equation does not specify the beta-function, but rather its derivative at the $g_{\mathrm{H}}=g_{\mathrm{H}}^{*}$ point. The reason is that our solution breaks down at energies $U \sim R^{2} / \rho_{0}$. From (21) we see that

$$
\beta^{\prime}\left(g_{\mathrm{H}}^{*}\right)=-4
$$


which means that $g_{\mathrm{YM}}^{*}$ is a UV-stable fixed point.f We believe that (22) is universal, namely, that it is valid for all models that approach $A d S_{5} \times S^{5}$ at some boundary. This can be seen by recalling that, near $A d S_{5} \times S^{5}$, the dilaton always satisfies (3) with $\Omega=R / \rho$. As a result, $\Phi$ will behave as $e^{\Phi-\Phi_{0}} \sim \rho^{4}$, where $\Phi_{0}$ is the value of $\Phi$ at $\rho=0$. We also see that (21) determines the second and third derivatives of the coupling beta-function at the fixed point, which, however, are not expected to be modelindependent. Let us also note that there is no known perturbative field theory with UV-stable fixed points. A behaviour of the form (20), namely power-law running of the couplings, was also found in type-0 theories (see second ref. in [6]), in gauge theories in higher dimensions [11] and extensively discussed in gauge-coupling unification in theories with large internal dimensions [12]-[15]. In this scenario, the internal dimensions are shown up in the four-dimensional theory as the massive KK modes. These modes can run in the loops of the four-dimensional theory, giving rise to a power law running of the couplings. In particular, for $d$ large extra dimensions and for energies $E$ above the infrared cutoff, which is specified by the mass scale $\mu$ of the extra dimensions, we find, just for dimensional reasons, that the running coupling constant of the effective fourdimensional theory is of the form $g^{(4)}-g_{0}^{(4)} \sim(\mu / E)^{d / 2}$, where $g_{0}^{(4)}$ is the bare coupling. Thus, in our case, since we have a four-dimensional theory coming from ten dimensions, we should expect the coupling to run in the sixth power of $E$. Instead, we find here that the coupling depends on the fourth power of $U=E$, indicating that when holography is involved, we get a softer running of the couplings.

It is possible to identify those operators that are responsible for the running of the coupling in the boundary field theory. Since the dilaton approaches a constant value at $\rho=0$ and the asymptotic background is an $A d S_{5}$ space, the corresponding boundary field theory is expected to be a deformation of the $\mathcal{N}=4 S U(N)$ supersymmetric YM theory. The explicit form of the deformation may be specified by recalling that our solution still has an $S O(6)$ symmetry. There are not many $S O(6)$ singlets in the spectrum of the $S^{5}$ compactification. In fact, from the results in [16] we see that the only scalar singlets are the complex scalar of type-IIB theory $B, a_{i j k l}$ and $h_{i}^{i}$, with masses $m_{B}^{2}=0, m_{a}^{2}=m_{h}^{2}=32$ in AdS-mass units. Since we have not perturbed the five-form $F_{5}, a_{i j k l}=0$ and thus the perturbations we have turned on are the real part of $B$ and $h_{i}^{i}$. From their masses we find that the former corresponds to marginal deformations of the type $F_{\mu \nu}^{2}$, while the latter corresponds, to the dimension-eight operator $F_{\mu \nu}^{4}$, which is irrelevant. However, it gives contributions to the boundary field theory since we have an IR cutoff specified by $\rho_{0}$. Sending $\rho_{0} \rightarrow \infty$ all bulk perturbations disappear and, similarly, the boundary field theory turns out to be the $\mathcal{N}=4$ large- $N S U(N)$ SYM theory.

\footnotetext{
${ }^{4}$ Using a radious/energy relation in horospherical coordinates of the form $U=R^{2} e^{-r / R}$ we find that the running of $g_{\mathrm{H}}$ is

$$
U \frac{d g_{\mathrm{H}}}{d U}=-a g_{\mathrm{H}}^{*}\left(\left(\frac{g_{\mathrm{H}}}{g_{\mathrm{H}}^{*}}\right)^{1+\frac{1}{a}}-\left(\frac{g_{\mathrm{H}}}{g_{\mathrm{H}}^{*}}\right)^{1-\frac{1}{a}}\right) .
$$

However, the above expression is trustable only around the fixed point $g_{\mathrm{H}}^{*}$.
} 


\section{The quark-antiquark potential}

The breaking of the superconformal invariance of the $\mathcal{N}=4$ theory by our solution should be apparent in the expression for the quark-antiquark potential, which we now compute along the lines of [17, [18]. We will find corrections to the purely Coulombic behaviour, which, on purely dimensional grounds, we expect to be in powers of $A L^{4}$, where $L$ is the quark-antiquark distance. We are eventually interested in the first such correction, which, as is apparent when comparing (14) with (12), is due to the dilaton, but for the moment we keep the formalism general. As usual, we have to minimize the Nambu-Goto action

$$
S=\frac{1}{2 \pi} \int d \tau d \sigma \sqrt{\operatorname{det}\left(G_{M N} \partial_{\alpha} X^{M} \partial_{\beta} X^{N}\right)},
$$

where $G_{M N}$ is the target-space metric in the string frame. For the static configuration $x^{0}=\tau, x^{1} \equiv x=\sigma$, and $x^{2}, x^{3}$ as well as the coordinates of $S^{5}$ held fixed, we find that (24) becomes (we use the notation of [17])

$$
S=\frac{T}{2 \pi} \int d x e^{\Theta / 2} \sqrt{\left(\partial_{x} U\right)^{2}+U^{4} / R^{4}},
$$

where $e^{\Theta} \equiv e^{\Phi} \Sigma^{4}$ and $\Sigma=1+\sum_{n=1}^{\infty} a_{n}\left(\frac{R^{2}}{\rho_{0} U}\right)^{8 n}$ is the function multiplying $R / \rho$ in (14) (rewritten using $\rho=R^{2} / U$ ). It is clear that any background that approaches $A d S_{5}$ will always have a $\Sigma$ of the form (14), so that our analysis is quite general at this point. It is easy to see that the solution is expressed as

$$
x=\int_{U_{0}}^{U} \frac{R^{2} d U}{U^{2} \sqrt{e^{\Theta-\Theta_{0}} U^{4} / U_{0}^{4}-1}},
$$

where $U_{0}$ is the smallest "distance" of the trajectory to the center and $\Theta_{0}$ is the value of the function $\Theta$ evaluated at $U_{0}$. We assume that one of the branes is taken out to $U=\infty$ and that the string configuration starts and ends at this brane. The rest of the branes are located at $U=R^{2} / \rho_{0}$. Setting $x=L / 2$ corresponds to $U=\infty$. In turn, this gives a condition that relates $U_{0}$ to $L$ as

$$
L=2 \int_{U_{0}}^{\infty} \frac{R^{2} d U}{U^{2} \sqrt{e^{\Theta-\Theta_{0}} U^{4} / U_{0}^{4}-1}} .
$$

Proceeding in a standard fashion, we substitute back our solution (26) into the action (25) and obtain an integral that is infinity. This is because we have included into the potential energy the (equal) masses of the infinitely heavy (in the supergravity approximation) quark and antiquark. In order to compute these masses, we assume that $N$-branes are at $U=R^{2} / \rho_{0}$ and 1 at $U=U_{\max }$ that is assumed to be large but finite. The mass of a single quark is computed if in (25) we consider a configuration with $x^{0}=\tau, U=\sigma$ and with fixed spatial world-volume coordinates $x^{\alpha}, \alpha=1,2,3$, as well as $S^{5}$ coordinates. Then the self-energy of the quark is

$$
E_{\text {self }}=\frac{1}{2 \pi} \int_{R^{2} / \rho_{0}}^{U_{\max }} d U e^{\Theta / 2}
$$


Subtracting off this energy twice and letting $U_{\max } \rightarrow \infty$, we obtain a finite result for the quark-antiquark potential given by

$$
E_{q \bar{q}}=\frac{1}{\pi} \int_{U_{0}}^{\infty} d U \frac{U^{2} / U_{0}^{4} e^{\Theta / 2}}{\sqrt{U^{4} / U_{0}^{2}-e^{\Theta}-\Theta}}-\frac{1}{\pi} \int_{R^{2} / \rho_{0}}^{\infty} d U e^{\Theta / 2}
$$

At this point we have to solve (27) for $U_{0}$ as a function of $L$ and substitute the result back into (29) to obtain $E_{q \bar{q}}$ as a function of $L$ only. This can be done perturbatively in powers of $L / \rho_{0}$, and we are interested in the first correction to the Coulombic law behaviour of the potential. As explained, the correction due to the non-constant dilaton is dominant and we will therefore use $\Omega=R / \rho$, which corresponds to $A d S_{5} \times S^{5}$ for the string metric, whereas for the string coupling we will keep the first two terms in (12). Then we find that

$$
U_{0} \simeq \frac{2 R^{2} \eta_{1}}{L}\left(1+\frac{s}{8}\left(\frac{\eta}{2 \eta_{1}}\right)^{2}\left(\frac{L}{\rho_{0}}\right)^{4}\right), \quad \eta_{1}=\frac{\pi^{1 / 2} \Gamma(3 / 4)}{\Gamma(1 / 4)} \simeq 0.599
$$

where the value of the numerical constant $\eta$ has been given in (9) and

$$
E_{\mathrm{self}} \simeq \frac{U_{\max }}{2 \pi}-\frac{R^{2}}{2 \pi \rho_{0}}\left(1-s \frac{\eta^{4}}{24}\right)
$$

The result for the quark-antiquark potential is

$$
E_{q \bar{q}} \simeq-\frac{2 \eta_{1}^{2} R^{2}}{\pi} \frac{1}{L}\left(1-\frac{s}{8}\left(\frac{\eta}{2 \eta_{1}}\right)^{4}\left(\frac{L}{\rho_{0}}\right)^{4}\right)+\frac{R^{2}}{\pi \rho_{0}}\left(1-s \frac{\eta^{4}}{24}\right) .
$$

We see that the Coulombic potential receives a correction proportional to $L^{3}$ due to the breaking of conformal invariance. This is remarkably similar to the potential obtained in 19] for the quark-antiquark pair for $\mathcal{N}=4$, at finite temperature, using the near-horizon supergravity solution for $N$ coincident $D 3$-branes. However, in that case supersymmetry is broken by thermal effects, whereas in our case it is broken, by the presence of a nontrivial dilaton, even at zero temperature. The last term in (32) does not depend on $L$ and represents a constant shift of the potential energy.

As a final remark we note that the computation of the potential for the monopoleantimonopole pair proceeds along the same lines as that for the quark-antiquark pair, with the only difference that we start, similarly to [20], with the action for a D-string. This means that the integrand in (25) should be multiplied by $e^{-\Phi}$. Hence, the function $e^{\Theta}$ entering into (25) is defined as $e^{\Theta}=e^{-\Phi} \Sigma^{4}$. Consequently, the first correction to the Coulombic behaviour of the monopole-antimonopole potential is given by

$$
E_{m \bar{m}} \simeq-\frac{2 \eta_{1}^{2} R^{2}}{\pi g_{s} L}\left(1+\frac{s}{8}\left(\frac{\eta}{2 \eta_{1}}\right)^{4}\left(\frac{L}{\rho_{0}}\right)^{4}\right)+\frac{R^{2}}{\pi \rho_{0}}\left(1+s \frac{\eta^{4}}{24}\right),
$$

\footnotetext{
${ }^{5}$ Supergravity is valid when $\rho \ll \rho_{0}$, which means that $U \geq U_{0} \gg R^{2} / \rho_{0}$. Using the leading term in (27), we deduce that $L / \rho_{0} \ll 1$, which is indeed the condition for the validity of $(32)$.
} 
which is the same as (32) after we use the fact that under S-duality $g_{s} \rightarrow 1 / g_{s}$ and $s \rightarrow-s$. Hence we see a screening (antiscreening) of the quark-antiquark pair for $s=+1(-1)$ and exactly the opposite behaviour for the monopole-antimonopole pair.

\section{Acknowledgements}

We would like to thank K. Dienes, E. Dudas, T. Gherghetta, A.A. Tseytlin and A. Zaffaroni for discussions.

\section{References}

[1] J.H. Schwarz, Nucl. Phys. B226 (1983) 269.

[2] J. Maldacena, Adv. Theor. Math. Phys. 2 (1998) 231, hep-th/9711200.

[3] S.S. Gubser, I.R. Klebanov and A.M. Polyakov, Phys. Lett. B428 (1998)105, hepth/9802109; E. Witten, Adv. Theor. Math. Phys. 2 (1998) 253, hep-th/9802150.

[4] I.R. Klebanov and A.A. Tseytlin, D-branes and dual gauge theories in type 0 strings, hep-th/9811035, Asymptotic freedom and infrared behaviour in the type 0 string approach to gauge theories, hep-th/9812089; A non-supersymmetric large- $N$ CFT from type 0 string theory, hep-th/9901101.

[5] J.A. Minahan, Glueball mass spectra and other issues for supergravity duals of QCD models, hep-th/9811156; Asymptotic freedom and confinement from type 0 string theory, hep-th/9902074.

[6] G. Ferretti and D. Martelli, On the construction of gauge theories from non-critical type 0 strings, hep-th/9811208; E. Alvárez and C. Gómez, Non-critical confining strings and the renormalization group, hep-th/9902012.

[7] J. Distler and F. Zamora, Non-supersymmetric conformal field theories from stable Anti-de sitter spaces, hep-th/9810206; L. Girardello, M. Petrini, M. Porrati and A. Zaffaroni, Novel local CFT and exact results on perturbations of $N=4$ super YangMills from AdS dynamics, hep-th/9810126; A. Karch, D. Lüst and A. Miemiec, New $N=1$ superconformal field theories and their supergravity description, hepth/9901041.

[8] M. Cederwall, U. Gran, M. Holm and B.E.W. Nilsson, Finite tensor deformations of supergravity solitons, hep-th/9812144.

[9] S. Nojiri and S.D. Odintsov, Phys. Lett. B449 (1999) 39, hep-th/9812017.

[10] L. Susskind and E. Witten, The holographic bound in Anti-de Sitter space, hepth/9805114; A.W. Peet and J. Polchinski, UV/IR relations in AdS dynamics, hepth/9809022. 
[11] T.R. Taylor and G. Veneziano, Phys. Lett. B212 (1988) 147.

[12] I. Antoniadis, Phys. Lett. B246 (1990) 377.

[13] E. Witten, Nucl. Phys. B471 (1996) 135, hep-th/9602070; J.D. Lykken, Phys. Rev. D54 (1996) 3693, hep-th/9603133.

[14] K.R. Dienes, E. Dudas and T. Gherghetta, Phys. Lett. B436 (1998) 55, hepph/9803466; Nucl. Phys. B537 (1999) 47, hep-ph/9806292.

[15] C. Bachas, JHEP 23 (1998) 9811, hep-ph/9807415.

[16] M Günaydin and N. Marcus, Class. Quant. Grav. 2 (1985) L11; H.J. Kim, L.J. Romans and P. van Nieuwenhuizen, Phys. Rev. D32 (1985) 389.

[17] J. Maldacena, Phys. Rev. Lett. 80 (1998) 4859, hep-th/9803002.

[18] S-J. Rey and J. Yee, Macroscopic strings as heavy quarks in large $N$ gauge theory and Anti-de-Sitter supergravity, hep-th/9803001.

[19] A. Brandhuber, N. Itzhaki, J. Sonnenschein and S. Yankielowicz, Phys. Lett. B434 (1998) 36, hep-th/9803137.

[20] D.J. Gross and H. Ooguri, Phys. Rev. D58 (1998) 106002, hep-th/9805129. 